\title{
Perfil de mortalidad en una Unidad de Cuidados Intensivos Pediátricos: asociación entre la mortalidad y el período fuera del horario diurno semanal
}

\author{
Six year mortality profile of a Pediatric Intensive Care Unit: \\ association between out-of-hours and mortality
}

\section{Dra. Ganime Ayar ${ }^{a}$, Dra. Mutlu Uysal Yazici ${ }^{b}$, Dra. Sanliay Sahin ${ }^{a}$, Dr. Ramiz C. Gunduza, Dr. Halil I. Yakut , Dra. Alkim Oden Akman y Dr. Gokhan Kalkan ${ }^{c}$}

\section{RESUMEN}

Introducción. El personal capacitado y los avances tecnológicos mejoraron las unidades de cuidados intensivos pediátricos (UCIP); por ende, mejoraron la atención, la supervivencia y el pronóstico. Pero la calidad de la atención varía según la disponibilidad de recursos humanos y técnicos.

Objetivo. Determinar la correlación de las tasas de mortalidad dentro y fuera del horario diurno en días de semana.

Métodos. Se definió horario de días de semana de 8:00 a. m. a 5:00 p. m., el período fuera del horario fueron de 5:00 p. m. a 8:00 a. m., fines de semana y feriados, con un residente en la UCIP y un médico de planta de guardia pasiva. Se clasificaron las causas de mortalidad en la UCIP. Resultados. Se hospitalizaron 2187 pacientes en la UCIP. Fallecieron 394; 151 niñas $(38,3 \%$ ) y 243 varones $(61,7 \%)$. Según el horario de muerte, la mayoría ocurrió fuera del horario diurno $244(61,9 \%)$ versus a $150(38,1 \%)$ durante el turno diurno, una diferencia estadísticamente significativa $(p<0,05)$. La edad, el sexo y la duración de la hospitalización en la UCIP no fueron significativos $(p>0,05)$ al comparar el período dentro y fuera del horario diurno. Se evaluaron las afecciones que acompañaron la muerte. La relación entre el horario de médicos de planta y la muerte fue significativa $(p<0,05)$. Hubo más afecciones asociadas a mortalidad fuera del horario diurno .

Conclusión. El período fuera del horario diurno sin médicos de planta estuvo asociado con mayor mortalidad.

Palabras clave: críticamente enfermo; niño; mortalidad; atención fuera del horario con médicos de planta; Unidad de Cuidado Intensivo Pediática.

http:/ / dx.doi.org/10.5546/aap.2019.120

Texto completo en inglés:

http:/ / dx.doi.org/10.5546/ aap.2019.eng.120

Conflicto de intereses:

Ninguno que declarar.

Recibido: 11-4-2018

Aceptado: 27-12-2018

Publicado Primero en Internet: 21-2-2019
Cómo citar: Ayar G, Uysal Yazici M, Sahin S, Gunduz RC, et al. Perfil de mortalidad en una Unidad de Cuidados Intensivos Pediátricos: asociación entre la mortalidad y el período fuera del horario diurno semanal. Arch Argent Pediatr 2019;117(2):120-125.

\section{INTRODUCCIÓN}

Las tasas de supervivencia han aumentado gradualmente y el pronóstico es mejor gracias al personal altamente capacitado y a la tecnología empleada en las unidades de cuidados intensivos pediátricos (UCIP), donde se atiende a pacientes críticos. ${ }^{1,2}$ Se requiere experiencia en la planificación de una UCIP en los países desarrollados. ${ }^{3}$ Recientemente, las tasas de mortalidad comenzaron a disminuir gracias a la mayor cantidad de personal con formación en el campo de los cuidados intensivos pediátricos. ${ }^{4,5} \mathrm{Se}$ brinda respiración mecánica a través de respiradores, además de muchos procedimientos médicos que salvan vidas, como peritoneo/hemodiálisis, plasmaféresis, oxigenación por membrana extracorporal y diversas cirugías. En la UCIP, las infecciones intrahospitalarias podrían llevar a una prolongación de la hospitalización, al aumento de los costos y a la resistencia a los antibióticos como ocurre en los adultos. ${ }^{6}$ Según los estudios disponibles, la mortalidad es mayor durante las hospitalizaciones nocturnas. ${ }^{7}$ En algunos estudios, se indica que el ingreso fuera del turno diurno no aumentaría la mortalidad ${ }^{8-10}$ y que el ingreso después del turno diurno estaría asociado con una reducción de la mortalidad.$^{11}$ Sin embargo, la cantidad de estudios que evaluaron los resultados de los pacientes hospitalizados en la UCIP son limitados. Presuntamente, 
existiría una diferencia entre los recursos humanos y profesionales disponibles durante los distintos turnos; el problema no sería solo una menor cantidad de ellos sino también su cansancio y otros factores que afectan la calidad de la atención. Por lo tanto, el objetivo del estudio fue determinar la correlación de las tasas de mortalidad entre el turno diurno y otros turnos (fuera del horario de planta) para contribuir a mejorar la calidad de la atención de los pacientes en estas unidades.

\section{MATERIAL Y MÉTODOS}

A los fines de este estudio, se revisaron retrospectivamente las historias clínicas de los niños hospitalizados consecutivamente en la UCIP durante seis años, entre diciembre de 2006 y diciembre de 2012, tras la aprobación del Comité de Ética institucional. La UCIP cuenta con 14 camas y se encuentra en un centro multidisciplinario de alta complejidad de referencia, donde se atienden entre 400 y 450 pacientes cada año. Allí se reciben pacientes de todo el país y el hospital funciona como un centro de referencia para enfermedades hematooncológicas donde se realizan trasplantes de médula ósea. En el estudio, se incluyó a todos los niños hospitalizados en la UCIP, independientemente de la enfermedad preexistente. La edad de los niños ingresados varió entre 40 días y 18 años. Se registraron como variables la edad, el sexo, el diagnóstico, la duración de la hospitalización, las comorbilidades y el momento de la muerte. Se agrupó a los pacientes según el sistema implicado con respecto a los problemas más importantes que podrían causar la muerte; se excluyeron las historias clínicas incompletas.

En esta unidad, trabajaban tres pediatras (dos capacitados/certificados especialmente en cuidados intensivos pediátricos), cuatro pediatras asistentes en investigación y seis enfermeros (con experiencia en la UCIP) durante el horario diurno. También hubo un especialista en cuidados intensivos pediátricos durante dos años del período del estudio. Durante la noche y los fines de semana, solo un pediatra asistente en investigación estaba en la unidad, junto con un asistente en investigación superior y un pediatra ( in experiencia en la UCIP), quienes eran responsables de todas las unidades del hospital, incluida la UCIP. La cantidad de enfermeros también disminuía durante los turnos nocturnos y fin de semana, de seis a cuatro. Se definió el horario diurno como días de semana de 8:00 a. m. a 5:00 p. m., con asistencia interna del médico de planta (senior staff) y el período fuera de ese horario se definió como días de semana de 5:00 p. m. a 8:00 a. m., fines de semana y feriados, con asistencia de un residente que cubre la UCIP y un médico de planta de guardia pasiva. Para los cálculos, asumimos que el personal trabajó todas las semanas del año sin tomarse vacaciones y que el horario diurno era de 8:00 a. m. a 5:00 p. m., cinco días a la semana, y que el resto de los horarios correspondían al período fuera de ese horario.

\section{Análisis estadístico}

Los datos se evaluaron con el programa SPSS (Statistical Package for Social Sciences) para Windows, versión 16. La normalidad de los datos se evaluó con la prueba de normalidad de Shapiro-Wilk. Las variables con una distribución normal se expresaron como media $\pm \mathrm{DE}$; aquellas con una distribución anormal, como mediana (mínimo-máximo). Se usó la prueba $t$ de Student para las variables con distribución normal; la prueba U de Mann-Whitney se empleó para comparar los grupos con variables cuya distribución no era normal. La prueba de $\chi^{2}$ se utilizó para comparar las variables categóricas. Un valor de $p<0,05$ se consideró significativo en términos estadísticos.

\section{RESULTADOS}

En total, 2187 niños ingresaron a la UCIP en un período de seis años. Se incluyeron en el estudio 394 pacientes que fallecieron allí; 151 eran niñas $(38,3 \%)$ y 243 , varones $(61,7 \%)$. Se excluyeron 6 pacientes porque sus historias clínicas estaban incompletas. La media de edad de los pacientes fue de 93,1 meses (mín.: 2 meses, máx.: 288 meses, mediana: 70,5 meses). La media de la hospitalización fue 9 días (mín.: 1 día, máx.: 79 días, mediana: 5 días).

Se analizaron el diagnóstico de los pacientes y la relación entre los horarios de trabajo y la mortalidad. La muerte ocurrió fuera del horario diurno en 244 pacientes $(61,9 \%)$ y dentro del horario con médico de planta, en 150 pacientes $(38,1 \%)$; la diferencia fue estadísticamente significativa $(p<0,05)$. Asimismo, la edad, el sexo y la duración de la hospitalización en la UCIP no fueron significativos $(p>0,05)$ en la comparación entre el período dentro y fuera del horario diurno Se registraron las afecciones que acompañaron la muerte y se evaluaron en 
TABla 1. Afecciones que acompañaron la muerte en la unidad de cuidados intensivos pediátricos

\begin{tabular}{|c|c|}
\hline Variables & n $(\%)$ \\
\hline Distribución de los diagnósticos & $685(100)$ \\
\hline Enfermedades respiratorias & $168(24,5)$ \\
\hline Neumonía & $129(18,8)$ \\
\hline Neumonía por aspiración & $13(1,9)$ \\
\hline Derrame pleural & $8(1,2)$ \\
\hline Neumonía por H1N1 & $7(1,0)$ \\
\hline Síndrome de dificultad respiratoria aguda & $7(1,0)$ \\
\hline Neumotórax & $4(0,6)$ \\
\hline Enfermedades infecciosas & $106(15,5)$ \\
\hline Sepsis & $69(10,1)$ \\
\hline Infección del SNC & $16(2,3)$ \\
\hline Gastroenteritis aguda & $8(1,2)$ \\
\hline Infección por citomegalovirus (CMV) & $6(0,9)$ \\
\hline Fiebre hemorrágica de Crimea y el Congo & $3(0,4)$ \\
\hline Tuberculosis & $2(0,3)$ \\
\hline Varicela & $2(0,3)$ \\
\hline Enfermedades hematológicas & $89(13,0)$ \\
\hline Anemia & $34(4,9)$ \\
\hline Leucemia mielógena aguda & $17(2,5)$ \\
\hline Leucemia linfocítica aguda & $12(1,8)$ \\
\hline Linfoma & $11(1,6)$ \\
\hline Coagulación intravascular diseminada & $10(1,5)$ \\
\hline Trombosis venosa profunda & $5(0,7)$ \\
\hline Eventos intracraneales & $81(11,8)$ \\
\hline Hemorragia intracraneal & $25(3,7)$ \\
\hline Masa intracraneal & $24(3,5)$ \\
\hline Anomalía del SNC & $14(2,0)$ \\
\hline Edema cerebral & $14(2,0)$ \\
\hline Hernia & $4(0,6)$ \\
\hline Enfermedades neurológicas & $77(11,2)$ \\
\hline Parálisis cerebral/retraso mental motor & $27(3,9)$ \\
\hline Hipotonía & $22(3,2)$ \\
\hline Atrofia muscular espinal & $14(2,1)$ \\
\hline Enfermedades neurodegenerativas & $7(1,0)$ \\
\hline Enfermedades mitocondriales & $7(1,0)$ \\
\hline Enfermedades cardíacas & $58(8,5)$ \\
\hline Cardiopatía congénita & $32(4,7)$ \\
\hline Hipertensión pulmonar & $8(1,2)$ \\
\hline Miocardiopatía hipertrófica & $8(1,2)$ \\
\hline Miocardiopatía dilatada & $7(1,0)$ \\
\hline Miocarditis & $3(0,4)$ \\
\hline Nefropatías & $46(6,7)$ \\
\hline Insuficiencia renal aguda & $38(5,6)$ \\
\hline Vasculitis & $5(0,7)$ \\
\hline Insuficiencia renal crónica & $3(0,4)$ \\
\hline Endocrinopatías & $10(1,5)$ \\
\hline Diabetes insípida & $4(0,6)$ \\
\hline Hipotiroidismo & $3(0,4)$ \\
\hline Insuficiencia suprarrenal & $2(0,3)$ \\
\hline Diabetes mellitus & $1(0,2)$ \\
\hline Enfermedades gastrointestinales & $36(5,3)$ \\
\hline Insuficiencia hepática & $21(3,1)$ \\
\hline Hemorragia gastrointestinal & $5(0,7)$ \\
\hline Gastroenteritis crónica & $5(0,7)$ \\
\hline Obstrucción intestinal & $3(0,4)$ \\
\hline Perforación intestinal & $1(0,2)$ \\
\hline Pancreatitis aguda & $1(0,2)$ \\
\hline Traumatismos & $14(2,0)$ \\
\hline Traumatismo craneoencefálico & $9(1,3)$ \\
\hline Traumatismo torácico & $3(0,4)$ \\
\hline Traumatismo penetrante & $2(0,3)$ \\
\hline
\end{tabular}

SNC: sistema nervioso central. conjunto; la única relación significativa fue entre los horarios evaluados y la mortalidad $(p<0,05)$. No hubo diferencias significativas en términos de diagnósticos acompañantes. Se observó una mayor cantidad de afecciones relacionadas con la mortalidad fuera del horario diurno. En la Tabla 1, se resumen los diagnósticos de las afecciones que acompañaron la muerte; en la Tabla 2, se detalla el perfil de mortalidad dentro y fuera del horario diurno en días de semana.

Las enfermedades hematológicas representaron un alto porcentaje en nuestro hospital, donde se controlan relativamente más pacientes con enfermedades hematooncológicas. También se observaron inmunodeficiencia primaria en 17 pacientes $(4,3 \%)$, inmunodeficiencia secundaria en $10(2,5 \%)$ y enfermedad injerto contra huésped (EICH) en 4 , quienes se sometieron a un trasplante de médula ósea. Se detectaron sepsis en 4 pacientes que tenían inmunodeficiencia primaria; infección por citomegalovirus (CMV), en 2; tuberculosis, en 1; e infección del sistema nervioso central, en 1. Entre 12 pacientes con micosis, en 2 se observó sepsis por Candida. Se registró aspergilosis pulmonar invasiva en 10 pacientes $(2,5 \%)$.

Se detectaron enfermedades metabólicas en 29 pacientes $(7,4 \%)$. Se observaron síndromes genéticos en 22 pacientes $(5,6 \%$ ) (síndrome de Down en 9; otros síndromes, en 13). Trece pacientes llegaron a la sala de emergencias con paro cardíaco y fallecieron en la UCIP. Además, 2 pacientes sufrieron muerte por inmersión; 1, intoxicación por monóxido de carbono; y 5 , intoxicación por diversos fármacos.

Se produjeron muertes por traumatismo solo en 14 pacientes en nuestro hospital, una cifra menor a la prevista dado que un hospital cercano funciona como un centro de traumatología. Se detectaron traumatismo craneoencefálico en 4 pacientes $(1 \%)$ y traumatismo generalizado en $10(2,5 \%)$. En 13 pacientes $(3,3 \%)$ se produjo muerte cerebral.

\section{DISCUSIÓN}

El objetivo de este estudio era demostrar la relación entre la mortalidad en el horario diurno versus el horario nocturno y los de fin de semana y feriados que tenían un entorno de recursos limitados. Creemos que la cantidad y la calidad de los dispositivos y del personal capacitado constituyen un factor importante que aumenta las tasas de supervivencia en la UCIP. Según los estudios que investigaron la influencia del 
horario de hospitalización sobre la mortalidad en la UCIP, los resultados son variados. En un estudio multicéntrico retrospectivo, de seis años de duración, realizado por Arias y col. en 15 centros, se demostró que la tasa de mortalidad fue mayor en los pacientes hospitalizados durante la noche y no se observó una diferencia significativa entre la tasa de mortalidad de los pacientes hospitalizados los días de semana y aquella correspondiente a los fines de semana. ${ }^{7}$ De manera similar a nuestros resultados, en un estudio realizado en los Estados Unidos, la tasa de mortalidad fue mayor fuera del horario diurno en comparación con el turno fuera de ese horario y los fines de semana. ${ }^{11}$ En varios estudios llevados a cabo en Turquía, Estados Unidos, Australia, Italia e Inglaterra, se demostró que el ingreso a la UCIP fuera del horario con médicos de planta no aumentó la mortalidad, mientras que el ingreso de los pacientes fuera de ese horario mostró una relación con una disminución de la mortalidad en un estudio en un solo centro realizado en Australia. ${ }^{8-12}$ En un estudio de Italia, la ausencia de una persona a cargo durante las 24 horas en la unidad no afectó la mortalidad. ${ }^{9}$ En nuestro estudio, se analizó el horario de la muerte en los pacientes que fallecieron en la UCIP dentro y fuera del horario diurno con la presencia de un médico de planta. No obstante, si bien los estudios encontrados en la bibliografía investigaron, en su mayoría, la influencia del horario de ingreso a la UCIP sobre la mortalidad, en nuestro estudio se analizó la relación con el horario del fallecimiento.

De manera que acorde con la bibliografía, en nuestro estudio, las enfermedades respiratorias fueron la causa de muerte más frecuente, ${ }^{1,4}$ seguidas de otras enfermedades infecciosas y de las enfermedades hematológicas. En el análisis del diagnóstico primario de los pacientes que fallecieron en la UCIP, se observó que la mayor mortalidad tenía relación con las neoplasias. ${ }^{13}$ En la mayoría de los estudios, se demostró que la sepsis era la causa de muerte más frecuente..$^{5,14}$ En nuestro estudio, la sepsis también fue la causa más común de infección secundaria. En un estudio realizado en pacientes que fallecieron en la UCIP en los Países Bajos, los diagnósticos notificados al momento del ingreso de los pacientes fueron enfermedades neurológicas, enfermedades respiratorias, enfermedades circulatorias y otras enfermedades. ${ }^{15}$ En otro estudio, se notificaron problemas respiratorios, neurológicos y circulatorios como los diagnósticos más frecuentes al momento del ingreso de los pacientes que fallecieron en la UCIP. ${ }^{16}$ Las infecciones intrahospitalarias produjeron una mayor morbimortalidad, una prolongación de la hospitalización, un aumento de los costos y de la resistencia a los antibióticos, tanto en los pacientes pediátricos como en los adultos. ${ }^{6}$ En estudios previos, se indicó una asociación entre la mortalidad y las enfermedades hematológicas, los problemas neurológicos preexistentes, las enfermedades cardíacas congénitas, las inmunodeficiencias, la insuficiencia renal y las infecciones intrahospitalarias. ${ }^{17-19}$

Las inmunodeficiencias -tanto primarias como secundarias- produjeron neumonía más grave,

TABLA 2. Perfil de mortalidad detallado fuera y dentro del horario diurno

\begin{tabular}{|c|c|c|c|c|}
\hline Variables & $\begin{array}{c}\text { Total } \\
(\mathrm{n}=394)\end{array}$ & $\begin{array}{l}\text { Fuera del } \\
\text { horario diurno } \\
(\mathrm{n}=244)\end{array}$ & $\begin{array}{l}\text { Dentro del } \\
\text { horario diurno } \\
(\mathrm{n}=150)\end{array}$ & Significancia \\
\hline Edad (meses), media \pm DE (mediana) & $93,1 \pm 69,2(70,5)$ & $95,4 \pm 69,8(72,5)$ & $89,3 \pm 68,1(67,5)$ & $p>0,05$ \\
\hline $\begin{array}{ll}\text { Sexo, } & \text { masculino, } \mathrm{n}(\%) \\
& \text { femenino, } \mathrm{n}(\%)\end{array}$ & $\begin{array}{l}243(61,7) \\
151(38,3)\end{array}$ & $\begin{array}{l}158(64,8) \\
86(35,2)\end{array}$ & $\begin{array}{l}85(56,7) \\
65(43,39)\end{array}$ & $p>0,05$ \\
\hline Hospitalización (días) media \pm DE (mediana) & $9,0 \pm 10,8(5)$ & $8,5 \pm 10,7(4)$ & $9,7 \pm 10,9(7)$ & $\mathrm{p}>0,05$ \\
\hline \multicolumn{5}{|c|}{ Afecciones que acompañaron la muerte en la UCIP, n (\%) } \\
\hline Enfermedades respiratorias & 168 & $94(38,5)$ & $74(49,3)$ & \\
\hline Enfermedades infecciosas & 106 & $69(28,3)$ & $37(24,7)$ & \\
\hline Enfermedades hematológicas & 89 & $52(21,3)$ & $37(24,7)$ & \\
\hline Eventos intracraneales & 81 & $44(18)$ & $37(24,7)$ & \\
\hline Enfermedades neurológicas & 77 & $43(17,6)$ & $34(22,7)$ & $p>0,05$ \\
\hline Enfermedades cardíacas & 58 & $36(14,8)$ & $22(14,7)$ & \\
\hline Nefropatías & 46 & $32(13,1)$ & $14(9,3)$ & \\
\hline Enfermedades gastrointestinales & 36 & $21(8,6)$ & $15(10)$ & \\
\hline Traumatismos & 14 & $14(5,7)$ & 0 & \\
\hline Endocrinopatías & 10 & $9(3,7)$ & $1(0,7)$ & \\
\hline
\end{tabular}

DE: desviación estándar; UCIP: unidad de cuidados intensivos pediátricos. 
prolongación de la hospitalización y aumento de la mortalidad. Se realizó un trasplante de médula ósea en 4 pacientes con inmunodeficiencia primaria que tuvieron enfermedad de injerto contra huésped.

En nuestro estudio, los lactantes con parálisis cerebral/retraso mental motor e hipotonía representaron el $18 \%$ de los pacientes fallecidos; se detectaron convulsiones en el $15 \%$ de estos. La neumonía por aspiración fue el problema más frecuente en los pacientes con una enfermedad neurológica preexistente. Esto puede ser debido a las infecciones respiratorias frecuentes como resultado de una disfunción de la deglución y a un aumento de las secreciones debido al uso de ciertos antiepilépticos; estas afecciones produjeron una extensión de la hospitalización y prolongación del uso del respirador, neumonía relacionada con el respirador y aumento de la mortalidad por escoliosis grave, contracturas y deformación torácica.

En nuestro estudio, hubo en total 2187 hospitalizaciones en seis años, y la tasa de mortalidad fue del $18 \%$. La tasa de mortalidad notificada en la UCIP en los países desarrollados es de aproximadamente el $5-10 \% ; 8-11,15,16,20$ y en otro estudio de Turquía, fue del 12,2-17,4\%. ${ }^{4}$ En nuestro estudio, se consideró que la mayor tasa de mortalidad se relacionaba con la característica de nuestra UCIP, a la que se deriva la proporción más alta de pacientes con enfermedad hematooncológica en estadio terminal, provenientes de todo el país. Se prevé que los perfiles de las enfermedades muestren algunas diferencias según las características regionales. Aún es inadecuada la cantidad de estudios que investigaron la mortalidad en la UCIP. Los autores reconocen que las limitaciones del estudio son su diseño con revisión retrospectiva de las historias clínicas y el hecho de haberse realizado en un solo centro. Sin embargo, consideran que el tamaño adecuado de la muestra y el período extenso del estudio (seis años) son las fortalezas de este estudio.

La duración de la hospitalización en la UCIP y la gravedad de la enfermedad no han cambiado en los últimos 10 años, aunque se detectó una reducción significativa en las tasas de mortalidad. No obstante, aumentó la proporción de discapacidad leve o grave en los supervivientes. Esta tendencia afecta a los pacientes y a sus familias y, en consecuencia, a toda la comunidad. ${ }^{21,22}$ Recientemente, se realizaron más estudios sobre la calidad y la confiabilidad de la atención de los pacientes, por lo que los especialistas en cuidados intensivos deberían mejorar sus conocimientos y esfuerzos para optimizar la calidad de la atención. ${ }^{23,24}$ Además, la tasa de mortalidad está estrechamente asociada con las instalaciones de la UCIP. Conforme a las pautas basadas en la evidencia, la supervisión de los procedimientos y la evaluación de los resultados del desempeño son fundamentalmente importantes para mejorar la calidad de la atención. Los servicios sanitarios deberían depender del trabajo en equipo, ser confiables, eficaces, centrados en el paciente, oportunos, adecuados y equitativos.

Una cantidad suficiente de equipos sanitarios debidamente capacitados, dispositivos técnicos adecuados, el control de las infecciones, la rehabilitación de los niños con anomalías neurológicas y genéticas, y la mejoría del respaldo y de la supervisión en los turnos fuera del horario diurno son factores importantes para mejorar la calidad y la supervivencia en las UCIP donde se atiende a niños críticamente enfermos.

\section{CONCLUSIÓN}

Conforme a nuestros resultados, el período fuera del horario diurno, sin la presencia de médicos de planta, estuvo asociado con una mortalidad mayor.

\section{REFERENCIAS}

1. Volakli E, Sdougka M, Tamiolaki M, Tsonidis C, et al. Demographic profile and outcome analysis of pediatric intensive care patients. Hipokratia.2011;15(4):316-22.

2. Ay B, Tutanç M, Yel S, Kaplan M, et al. Yoğun Bakıma Yatan Hastalarda AT-III Seviyesi ve Prognostik Değeri. Mustafa Kemal Üniversitesi Tıp Dergisi. 2010;1(1):1-5.

3. Khilnani P, Sarma D, Singh R, Uttam R, et al. Demographic profile and outcome analysis of a tertiary level pediatric intensive care unit. Indian J Pediatr. 2004;71(7):587-91.

4. Aşılıŏlu N, Kot H. Çocuk Yoğun Bakım Ünitesine Yatan Olguların Değerlendirilmesi ve Sonuçları. Turkiye Klinikleri J Pediatr. 2011;20(1):10-5.

5. Tutanç M, Arıca V, Başarslan F, Karcıoğlu M, et al. Çocuk yoğun bakım ünitesine yatan hastaların değerlendirilmesi. Dïzce tıp Dergisi. 2011;13(3):18-22.

6. Eroğlu C. Hastane infeksiyonlar1. Infeksiyon. 2001;135-45.

7. Arias Y, Taylor DS, Marcin JP. Association between evening admissions and higher mortality rates in the pediatric intensive care unit. Pediatrics. 2004;113(6):e530-4.

8. Numa A, Williams G, Awad J, Duffy B. After-hours admissions are not associated with increased risk-adjusted mortality in pediatric intensive care. Intensive Care Med. 2008;34(1):148-51.

9. Peeters B, Jansen NJ, Bollen CW, van Vaught AJ, et al. Offhours admission and mortality in two pediatric intensive care units without 24-h in-house senior staff attendance. Intensive Care Med. 2010;36(11):1923-7. 
10. McShaneP,DraperES,McKinney PA, McFadzeanJ, Parslow RC. Effects of Out-of- Hours and Winter Admissions and Number of Patients per Unit on Mortality in Pediatric İntensive Care. J Pediatr. 2013;163(4):1039-44.e5.

11. Hixson ED, Davis S, Morris S, Harrison AM. Do weekends or evenings matter in a pediatric intensive care unit? Pediatr Crit Care Med. 2005;6(5):523-30.

12. Arslankoylu AE, Bayrakçı B, Oymak Y. Admission Time and Mortality Rates. Indian J Pediatr. 2008;75(7):691-4.

13. LanetzskiS, de Oliveira CA, Bass LM, AbramoviciS, Troster EJ. The epidemioligical profile of Pediatric İntensive Care Center at Hospital Israelita Albert Einstein. Einstein (San Paulo). 2012;10(1):16-21.

14. Matics TJ, Sanchez-Pinto LN. Adaptation and Validation of a Pediatric Sequential Organ Failure Assessment Score and Evaluation of the Sepsis-3 Definitions in Critically Ill Children. JAMA Pediatr. 2017;171(10):e172352.

15. ten Berge J, de Gast-Bakker DA, Plötz FB. Circumstances surrounding dying in the paediatric intensive care Unit. BMC Pediatr. 2006;6:22.

16. Gregherehchi R, Rafeey M. Prediction of Mortality Circumtances in the Pediatric İntensive Care Unit. Res J Biol Sci. 2008;3(9):1034-6.

17. Earle M Jr, Martinez Natera O, Zaslavsky A, Quinones E, et al. Outcome of pediatric intensive care at six centers in Mexico and Ecuador. Crit Care Med. 1997;25(9)1462-7.
18. Bailey D, Phan V, Litalien C, Ducruet T, et al. Risk factors of acute renal failure in critical ill children: A prospective descriptive epidemiological study. Pediatr Crit Care Med. 2007;8(1):29-35.

19. Goodman S, Shirov T, Weissman C. Supraventricular arrhythmias in intensive care unit patients: short and longterm consequences. Anesth Analg. 2007;104(4):880-6.

20. Jones S, Rantell K, Stevens K, Colwell B, et al. Outcome at 6 months after admission for pediatric intensive care: a report of a national study of pediatric intensive care units in the United kingdom. Pediatrics. 2006;118(5):2101-8.

21. Namachivayam P, Shann F, Shekerdemian L, Taylor A, et al. Three decades of pediatric intensive care: Who was admitted, what happened in intensive care, and what happened afterward. Pediatr Crit Care Med. 2010;11(5):54955.

22. Garros D, Rosychuk RJ, Cox PN. Circumstances surrounding end of life in a pediatric intensive care unit. Pediatrics. 2003;112(5):e371.

23. Chelluri LP. Quality and performance improvement in critical care. Indian I Crit Care Med. 2008;12(2):67-76.

24. López-Herce J, Del Castillo J, Matamoros M, Cañadas S, et al. Factors associated with mortality in pediatric in-hospital cardiac arrest: a prospective multicenter multinational observational study. Intensive Care Med. 2013;39(2):309-18.

\section{Obituario}

\section{Dr. Horacio Repetto}

Falleció el 7 de enero de 2019.

La ida de Horacio ha sido una gran pérdida para todos aquellos que lo conocimos en las instituciones que estuvo. Se destacó mucho en la Nefrología y también en la Pediatría General siendo el Jefe del Departamento de Pediatría del Hospital Alejandro Posadas, designado en 1987.

Desempeñó elevadas funciones en nuestra Sociedad de Pediatría, fue co-fundador del Comité de Nefrología en 1974 y secretario entre 1976 a 1978, y posteriormente Editor Jefe de Archivos Argentinos de Pediatría, desde 1997 a 2000. Asimismo, fue miembro fundador de la Asociación Latinoamericana de Nefrología Pediátrica en 1981 y consejero de la International Pediatric Nephrology Association.

Su gran bonhomía y humildad, estuvieron siempre presentes y así, tuvo el afecto de todos los que tuvieron el placer de haberlo conocido y sin duda, cada uno lo tendrá en su memoria. 